\title{
Front Matter: Volume 6655
}

, "Front Matter: Volume 6655," Proc. SPIE 6655, Organic Light Emitting Materials and Devices XI, 665501 (26 November 2007); doi:

10.1117/12.773914

SPIE Event: Photonic Devices + Applications, 2007, San Diego, California, United SPIE. States 


\section{PROCEEDINGS OF SPIE}

\section{Organic Light Emitting Materials and Devices XI}

Zakya H. Kafafi

Franky So

Editors

26-29 August 2007

San Diego, California, USA

Sponsored and Published by

SPIE

Volume 6655 
The papers included in this volume were part of the technical conference cited on the cover and title page. Papers were selected and subject to review by the editors and conference program committee. Some conference presentations may not be available for publication. The papers published in these proceedings reflect the work and thoughts of the authors and are published herein as submitted. The publisher is not responsible for the validity of the information or for any outcomes resulting from reliance thereon.

Please use the following format to cite material from this book:

Author(s), "Title of Paper," in Organic Light Emitting Materials and Devices XI, edited by

Zakya H. Kafafi, Franky So, Proceedings of SPIE Vol. 6655 (SPIE, Bellingham, WA, 2007) Article CID Number.

ISSN 0277-786X

ISBN 9780819468031

Published by

SPIE

P.O. Box 10, Bellingham, Washington $98227-0010$ USA

Telephone +1 3606763290 (Pacific Time) · Fax +1 3606471445

SPIE.org

Copyright (c) 2007, Society of Photo-Optical Instrumentation Engineers

Copying of material in this book for internal or personal use, or for the internal or personal use of specific clients, beyond the fair use provisions granted by the U.S. Copyright Law is authorized by SPIE subject to payment of copying fees. The Transactional Reporting Service base fee for this volume is $\$ 18.00$ per article (or portion thereof), which should be paid directly to the Copyright Clearance Center (CCC), 222 Rosewood Drive, Danvers, MA 01923. Payment may also be made electronically through CCC Online at copyright.com. Other copying for republication, resale, advertising or promotion, or any form of systematic or multiple reproduction of any material in this book is prohibited except with permission in writing from the publisher. The CCC fee code is $0277-786 \mathrm{X} / 07 / \$ 18.00$.

Printed in the United States of America.

Publication of record for individual papers is online in the SPIE Digital Library.

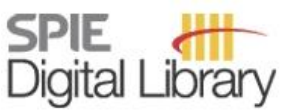

SPIEDigitalLibrary.org

Paper Numbering: Proceedings of SPIE follow an e-First publication model, with papers published first online and then in print and on CD-ROM. Papers are published as they are submitted and meet publication criteria. A unique, consistent, permanent citation identifier (CID) number is assigned to each article at the time of the first publication. Utilization of CIDs allows articles to be fully citable as soon they are published online, and connects the same identifier to all online, print, and electronic versions of the publication. SPIE uses a six-digit CID article numbering system in which:

- The first four digits correspond to the SPIE volume number.

- The last two digits indicate publication order within the volume using a Base 36 numbering system employing both numerals and letters. These two-number sets start with 00, 01, 02, 03, 04, 05, $06,07,08,09,0 \mathrm{~A}, 0 \mathrm{~B} \ldots \mathrm{OZ}$, followed by $10-1 \mathrm{Z}, 20-2 \mathrm{Z}$, etc.

The CID number appears on each page of the manuscript. The complete citation is used on the first page, and an abbreviated version on subsequent pages. Numbers in the index correspond to the last two digits of the six-digit CID number. 


\section{Contents}

ix Conference Committee

\section{SESSION 1 OLED MATERIALS I}

665502 Recent progress in deep-blue phosphorescent OLEDs (Invited Paper) [6655-05]

C. Schildknecht, C. Lennartz, K. Kahle, O. Molt, E. Fuchs, N. Moonen, J. Rudolph, G. Wagenblast, P. Amrhein, J. Proelss, I. Muenster, Joint Innovation Lab., BASF AG (Germany)

665504 Origin of the different emission wavelengths in Alq ${ }_{3}$ analyzed by solid-state NMR (Invited Paper) [6655-03]

H. Kaji, T. Fukushima, K. Takami, Y. Kusaka, Kyoto Univ. (Japan)

665505 Tuning the properties of polythienothiophene conductive polymers for hole injection layer application (Invited Paper) [6655-75]

X. Jiang, K. Campbell, F. Amy, B. Han, Air Products and Chemicals, Inc. (USA); F. So,

K. R. Choudhury, A. Gupta, Univ. of Florida (USA)

\section{SESSION 2 OLED MATERIALS II}

665506 Designing organic phosphine oxide host materials using heteroarmatic building blocks: inductive effects on electroluminescence (Invited Paper) [6655-06]

L. S. Sapochak, A. B. Padmaperuma, P. A. Vecchi, X. Cai, P. E. Burrows, Pacific Northwest National Lab. (USA)

665507 Synthesis and electroluminescent properties of poly( $p$-phenylene-vinylene)s with 3', 3'diheptyl-3,4-propylenedioxythiophene pendant group for light-emitting diode applications (Invited Paper) [6655-01]

S.-H. Jin, Pusan National Univ. (South Korea)

665508 New hole-transporting amorphous molecular materials with high glass-transition temperatures for organic light-emitting diodes (Invited Paper) [6655-08]

K. Okumoto, H. Doi, H. Kageyama, Osaka Univ. (Japan); Y. Shirota, Osaka Univ. (Japan) and Fukui Univ. of Technology (Japan)

665509 Organic materials for blue emission OLEDs (Invited Paper) [6655-09]

J. Shi, E. W. Forsythe, D. C. Morton, K. Dedeian, D. Chiu, S. M. Blomquist, U.S. Army Research Lab. (USA)

6655 OA Efficient blue organic light-emitting diodes with 4H-cyclopenta[def]phenanthrene [6655-10] H. Suh, Y. Jin, S. Song, C. Yoo, J. Kim, Pusan National Univ. (South Korea) 
6655 OC Emission and degradation mechanism of PLED (Invited Paper) [6655-38]

M. Koden, Sharp Corp. (Japan) and Nara Institute of Science and Technology (Japan); Y. Ohnishi, M. Nishimura, Nara Institute of Science and Technology (Japan); H. Uchida, Sharp Corp. (Japan)

\section{SESSION 4 TRIPLET EMITTERS}

6655 OF Spin-orbit coupling routes and OLED performance: studies of blue-light emitting Ir(III) and Pt (II) complexes (Invited Paper) [6655-11]

A. F. Rausch, H. H. H. Homeier, Univ. Regensburg (Germany); P. I. Djurovich, M. E. Thompson, Univ. of Southern California (USA); H. Yersin, Univ. Regensburg (Germany)

6655 OG Phosphorescent OLEDs with saturated colors (Invited Paper) [6655-14]

B. D'Andrade, V. I. Adamovich, M. S. Weaver, C. Lin, B. Ma, P. B. Mackenzie, R. Kwong, J. J. Brown, Universal Display Corp. (USA)

6655 Ol Harvest of triplet excitons in fluorescence emission layer based on a wide band gap host of TcTa for efficient white organic light emitting diodes [6655-13]

J.-I. Lee, H. Y. Chu, Y. S. Yang, L.-M. Do, S. M. Chung, S.-H. K. Park, C.-S. Hwang, Electronics and Telecommunications Research Institute (South Korea)

$66550 \mathrm{~J} \quad$ Harvesting triplet excitons from fluorescent blue emitters for high-efficiency white organic light emitting diodes [6655-70]

G. Schwartz, M. Pfeiffer, K. Walzer, K. Leo, Technische Univ. Dresden (Germany)

\section{SESSION 5 OLEDS AND FABRICATION PROCESSES}

6655 OK Spectrally narrowed edge emission from leaky waveguide modes in OLEDs (Invited Paper) [6655-15]

Z. Q. Gan, Y. Tian, D. W. Lynch, lowa State Univ. (USA); J. Kang, Q-H. Park, Korea Univ. (South Korea); J. Shinar, lowa State Univ. (USA)

6655 OL Highly efficient fully transparent inverted OLEDs [6655-16] J. Meyer, T. Winkler, S. Hamwi, S. Schmale, M. Kröger, P. Görrn, H.-H. Johannes, T. Riedl, Technische Univ. Braunschweig (Germany); E. Lang, D. Becker, T. Dobbertin, Osram Opto Semiconductors (Germany); W. Kowalsky, Technische Univ. Braunschweig (Germany)

$66550 \mathrm{M}$ Fabrication of multi-layered polymer LEDs by resonant infrared pulsed-laser deposition [6655-17]

S. L. Johnson, Vanderbilt Univ. (USA); H. K. Park, AppliFlex, LLC (USA); R. F. Haglund, Jr., Vanderbilt Univ. (USA)

6655 ON Technical issues of stainless steel foil substrates for OLED display applications (Invited Paper) [6655-18]

Y. Hong, S. Chung, Seoul National Univ. (South Korea); A. Kattamis, I-C. Cheng, S. Wagner, Princeton Univ. (USA) 
6655 OR Strong exciton-photon coupling in thermally evaporated polycrystalline organic materials (Invited Paper) [6655-40]

R. J. Holmes, Univ. of Minnesota (USA)

SESSION 7 ORGANIC LASERS

6655 OT Loss processes in organic double-heterostructure laser diodes [6655-26]

C. Gärtner, C. Karnutsch, J. Brückner, N. Christ, S. Uebe, U. Lemmer, Univ. Karlsruhe (TH)

(Germany); P. Görrn, T. Rabe, T. Riedl, W. Kowalsky, Technische Univ. Braunschweig (Germany)

6655 OV Polymer lasers: recent advances (Invited Paper) [6655-29]

T. Riedl, T. Rabe, P. Görrn, Technische Univ. Braunschweig (Germany); J. Wang,

T. Weinmann, P. Hinze, Physikalisch Technische Bundesanstalt (Germany); F. Galbrecht, U. Scherf, Bergische Univ. Wuppertal (Germany); W. Kowalsky, Technische Univ. Braunschweig (Germany)

6655 OW Diode-pumped polymer lasers [6655-30]

G. A. Turnbull, A. E. Vasdekis, G. Tsiminis, I. D. W. Samuel, Univ. of St. Andrews (United Kingdom)

SESSION 8 OLEDS AND SOLID STATE LIGHTING: JOINT SESSION WITH CONFERENCE 6669

6655 OX Employing microcavity effects to enhance performances of white-emitting OLEDs (Invited Paper) [6655-31]

C.-C. WU, Y.-J. LU, T.-Y. Cho, C.-L. Lin, C.-H. Chang, National Taiwan Univ. (Taiwan); L.-J. Chen, H.-L. Hsu, Y.-T. Lin, R. Nishikawa, TPO Displays Corp. (China)

6655 OY Progress in wet-coated organic light-emitting devices for lighting (Invited Paper) [6655-32] J. Liu, Q. Ye, L. N. Lewis, A. R. Duggal, General Electric Global Research (USA)

\section{SESSION 9 OLED LIGHTING}

665510 Improving the light extraction efficiency of polymer LEDs using microcavities and photonic crystals (Invited Paper) [6655-34]

A. M. Adawi, The Univ. of Sheffield (United Kingdom); M. Roberts, Cambridge Display Technology (United Kingdom); L. G. Connolly, R. Kullock, J. L. Turner, The Univ. of Sheffield (United Kingdom); E. Smith, C. Foden, F. Qureshi, N. Athanassopoulou, Cambridge Display Technology (United Kingdom); D. M. Whittaker, D. G. Lidzey, The Univ. of Sheffield (United Kingdom)

665511 Concepts for high efficient white OLEDs for lighting applications (Invited Paper) [6655-35] A. Hunze, Siemens AG (Germany); R. Krause, S. Seidel, Siemens AG (Germany) and Univ. of Erlangen-Nuremberg (Germany); O. Weiss, Siemens AG (Germany) and Technical Univ. of Darmstadt (Germany); F. Kozlowski, G. Schmid, Siemens AG (Germany); J. Meyer, M. Kröger, H. H. Johannes, W. Kowalsky, Technische Univ. Braunschweig (Germany);

T. Dobbertin, Osram Opto Semiconductors GmbH (Germany) 
665512 White phosphorescent organic light emitting devices [6655-36]

B. D'Andrade, M. S. Weaver, J. J. Brown, Universal Display Corp. (USA)

665513 Light extraction for a doubly resonant cavity organic LED: the RC2LED [6655-37]

P. Vandersteegen, Univ. Gent, IMEC (Belgium); S. Mladenovski, Univ. Gent (Belgium);

V. van Elsbergen, G. Gartner, Philips Technologie GmbH Forschungslaboratorien

(Germany); P. Bienstman, Univ. Gent, IMEC (Belgium); K. Neyts, Univ. Gent (Belgium);

R. Baets, Univ. Gent, IMEC (Belgium)

\section{SESSION 10 LIGHT-EMISSION IN ORGANIC STRUCTURES AND FIELD-EFFECT TRANSISTORS}

665514 Influence of the dielectric and of the active layer doping on the FET mobility in PPV-based devices (Invited Paper) [6655-07]

F. Todescato, R. Capelli, Istituto per lo Studio dei Materiali Nanostrutturati, CNR (Italy);

F. Dinelli, Istituto per i Processi Chimico Fisici, CNR (Italy); M. Murgia, Istituto per lo Studio dei Materiali Nanostrutturati, CNR (Italy); N. Camaioni, Istituto per la sintesi organica e la Fotoreattività, CNR (Italy); M. Yang, Zhejiang Univ. (China); M. Muccini, Istituto per lo Studio dei Materiali Nanostrutturati, CNR (Italy)

SESSION 11 CHARGE INJECTION AND TRANSPORT IN ORGANIC DEVICES: JOINT SESSION WITH CONFERENCE 6656

665519 The role of isoelectronic dopants in organic light emitting diodes (Invited Paper) [6655-43]

B. K. Crone, I. H. Campbell, D. L. Smith, Los Alamos National Lab. (USA)

SESSION 12 INTERFACES IN ORGANIC DEVICES: JOINT SESSION WITH CONFERENCE 6656

6655 ID Enhanced electron injection and performance in organic light emitting devices by Lif doping [6655-44]

K. R. Choudhury, J.-H. Yoon, F. So, Univ. of Florida (USA)

6655 IE Self-assembled monolayer modification of PEDOT:PSS interface to improve the device performance in blue PLED [6655-45]

Y. K. Lee, T. J. Park, W. S. Jeon, J. J. Park, J. H. Kwon, J. Jang, Kyung Hee Univ. (South Korea)

\section{POSTER SESSION}

$6655 \mathrm{IF}$ Optical gain in Coumarin 545T-doped Tris(8-hydroxy-chinolinato)aluminium thin films [6655-28]

T. Rabe, P. Görrn, T. Riedl, W. Kowalsky, Technische Univ. Braunschweig (Germany)

$66551 G$ White light-emitting organic electroluminescent device based on a new orange organometallic iridium complexes [6655-46]

T. Shieh, H. Huang, P. Liu, M.-R. Tseng, J.-M. Liu, Industrial Technology Research Institute (Taiwan) 
$66551 \mathrm{H}$ Optical characteristics of the OLED with microlens array film attachment [6655-49] H.-Y. Lin, J.-H. Lee, National Taiwan Univ. (Taiwan); M.-K. Wei, National Dong Hwa Univ. (Taiwan); K.-Y. Chen, S.-C. Hsu, Y.-H. Ho, C.-Y. Lin, National Taiwan Univ. (Taiwan)

665511 Oscillation method for uniform formation of solution-processed organic films and its application to organic light-emitting devices [6655-50]

T. Kitano, S. Naka, M. Shibata, H. Okada, Univ. of Toyama (Japan)

$66551 \mathrm{~J}$ Influence of cavity effects on light extraction efficiency in organic light emitting devices [6655-51]

J. Lee, N. Chopra, K. R. Choudhury, F. So, Univ. of Florida (USA)

$6655 \mathrm{lL}$ Lasing characteristics of optically pumped edge-emitting organic semiconductor laser [6655-53]

H. Yamaoka, M. Shibamoto, N. S. Takahashi, Keio Univ. (Japan)

$66551 \mathrm{M} \quad$ New conjugated polymer (PININE) with stability for LEDs [6655-54]

S. Song, Y. Jin, K. Kim, J. Kim, Pusan National Univ. (South Korea); J. Kim, S. Park, S. H. Kim, K. Lee, Gwangju Institute of Science and Technology (South Korea); H. Suh, Pusan National Univ. (South Korea)

$66551 \mathrm{~N} \quad$ Exclusive inkjet printed poly(3,4-ethylenedioxythiophene): polystyrenesulfonate as anode in polymer light-emitting diodes [6655-55]

S.-T. Lin, M.-H. Chang, J.-B. Horng, H.-L. Cheng, W.-Y. Chou, National Cheng Kung Univ. (Taiwan)

665510 A gas barrier film composed of $\mathrm{SiO}_{2} / \mathrm{Al}_{2} \mathrm{O}_{3}$ multilayers on flexible substrates [6655-56] J.-Y. Liao, P.-C. Liu, Y.-H. Yeh, M.-R. Tseng, Industrial Technology Research Institute (Taiwan)

6655 IP Studies of blue organic electroluminescent devices using the polymer/dopant systems as a light-emitting layer [6655-57]

F.-C. Wu, H.-L. Cheng, W.-Y. Chou, National Cheng Kung Univ. (Taiwan)

$66551 Q$ Carrier injection and bipolar transport in NPB for single-layer OLEDs [6655-58]

S. C. Tse, K. K. Tsung, S. K. So, Hong Kong Baptist Univ. (Hong Kong China)

6655 is Numerical simulation of top-emitting organic light-emitting diodes with electron and hole blocking layers [6655-60]

S.-H. Chang, C.-H. Yang, National Changhua Univ. of Education (Taiwan)

6655 IT Numerical simulation of bright white multilayer organic light-emitting diodes [6655-61] M.-L. Chen, C.-H. Yang, C.-Y. Wen, S.-H. Chang, Y.-K. Kuo, National Changhua Univ. of Education (Taiwan)

$66551 \mathrm{U}$ Light emission optimization of europium based complex in multilayer organic light emitting diodes [6655-62]

G. Santos, F. J. Fonseca, A. M. Andrade, Univ. de São Paulo (Brazil); M. Peres, T. Monteiro,

W. Simões, L. Pereira, Univ. de Aveiro (Portugal) 
$66551 \mathrm{~V}$ Electro-optical measurements, stability, and physical charge behavior of rare-earth based organic light emitting diode [6655-63]

G. Santos, F. J. Fonseca, A. M. Andrade, Univ. de São Paulo (Brazil); V. Deichmann, L. Ackcelrud, Univ. Federal do Paraná (Brazil); M. Peres, T. Monteiro, W. Simões, L. Pereira, Univ. de Aveiro (Portugal)

6655 IW Improved lifetime and efficiency of green organic light-emitting diodes with a fluorescent dye (C545T)-doped hole transport layer [6655-64]

H.-S. Bang, J. Yun, C. Lee, Seoul National Univ. (South Korea)

$66551 \mathrm{X}$ Tunable organic solid-state DFB laser utilizing molecular reorientation [6655-65]

H. C. Cheng, H. W. Lin, C. C. Wu, K. T. Wong, C. H. Kuan, National Taiwan Univ. (Taiwan)

Author Index 


\title{
Conference Committee
}

\author{
Symposium Chair
}

Zakya H. Kafafi, Naval Research Laboratory (USA)

Conference Chair

Zakya H. Kafafi, Naval Research Laboratory (USA)

Conference Cochair

Franky So, University of Florida (USA)

Program Committee

Chihaya Adachi, Kyushu University (Japan)

Alasdair J. Campbell, Imperial College London (United Kingdom)

Brian W. D'Andrade, Universal Display Corporation (USA)

Anil R. Duggal, General Electric Global Research (USA)

Tukaram K. Hatwar, Eastman Kodak Company (USA)

Andrew B. Holmes, The University of Melbourne (Australia)

Ghassan E. Jabbour, Arizona State University (USA)

Junji Kido, Yamagata University (Japan)

Changhee Lee, Seoul National University (South Korea)

Chun S. Lee, City University of Hong Kong (Hong Kong China)

Michele Muccini, Istituto per lo Studio dei Materiali Nanostrutturati , CNR (Italy)

Ifor D. W. Samuel, University of St. Andrews (United Kingdom)

Joseph Shinar, lowa State University (USA)

\section{Session Chairs}

1 OLED Materials I

Zakya H. Kafafi, Naval Research Laboratory (USA)

2 OLED Materials II

Franky So, University of Florida (USA)

3 Stability Issues in OLEDs

Graham A. Turnbull, University of St. Andrews (United Kingdom)

4 Triplet Emitters

Linda S. Sapochak, Pacific Northwest National Laboratory (USA) 
$5 \quad$ OLEDs and Fabrication Processes

Chihaya Adachi, Kyushu University (Japan)

$6 \quad$ Novel Light Emitting Structures and Devices

Michele Muccini, Istituto per lo Studio dei Materiali Nanostrutturati , CNR (Italy)

$7 \quad$ Organic Lasers

Brian K. Crone, Los Alamos National Laboratory (USA)

8 OLEDs and Solid State Lighting: Joint Session with Conference 6669

Hideyuki Murata, Japan Advanced Institute of Science and Technology (Japan)

$9 \quad$ OLED Lighting

Jie Liu, General Electric Global Research (USA)

10 Light-Emission in Organic Structures and Field-Effect Transistors

Chung-Chih Wu, National Taiwan University (Taiwan)

11 Charge Injection and Transport in Organic Devices: Joint Session with Conference 6656

Kwanghee Lee, Gwangju Institute of Science and Technology (South Korea)

12 Interfaces in Organic Devices: Joint Session with Conference 6656

Mark A. Baldo, Massachusetts Institute of Technology (USA) 